\title{
Research on Device Control System Design Based on Stepping Motor
}

\author{
Zhongyong Tian \\ The people's Hospital of Zhengzhou, Henan, Zhengzhou450003 \\ hunter2011@foxmail.com
}

Keywords: Stepping motor, Motion controller, Singlechip, The upper computer, Serial communication.

\begin{abstract}
Applying stepping motor to motion control system of equipment can make the device achieve the objective of two-dimensional motion control. Firstly, the paper defines the motion control scheme of system. Then, the paper makes detailed analysis on central processing unit and motion controller of hardware in system, and analyzes the software. The paper takes medical facility as an example. And the test proves that the system works well, which accurately implements the automatic positioning functions including corotation, reversal, acceleration and deceleration and pause, and achieves the design requirements.
\end{abstract}

\section{System Development Background}

As a digital servo executive component, stepping motor has the advantages of easy structure, reliable operation, easy control and good control performance, so stepping motor is widely applied in low-end market as the motion control system of executive component.

A medical facility is applied to make test analysis on the features of a medical reagent. The appliance consisting of test tube placing stage, screw and two stepping motors is placed in a seal box. The top of the box is fixed by a titration tube titrating reagent, and connects with external reagent bottle and the control circuit. The test tube placing stage is used to place the test tube vertically, in which $12 \times 8$ cylindrical grooves are distributed. Two stepping motors connects with test tube placing state with the structure of screw, and are used to control test tube placing state to move in the direction of $\mathrm{X}$ and $\mathrm{Y}$. And the tested solution of $12 \times 8$ test tubes is titrated orderly. And controlling the movement of the test tube placing can make it open the box door, which is easy for operators to take out or place in test tube. When test tube placing station moves in the direction of $\mathrm{Y}$, the stepping motor and the screw in direction of $\mathrm{X}$ moves. When it moves in the direction of $\mathrm{X}$, only test tube placing stage moves, and the stepping motor indirection $\mathrm{X}$ has no displacement in direction of $\mathrm{X}$. In order to ensure that the tested solution in testing tube and titration react normally under the given temperature, the temperature in the seal box should be constant. There is a door on the side of the box, which can control test placing stage in or out of calorstat, which makes it convenient for operators to place test tubes.

The paper researches tow-axle stepping motor motion controller, and the controller is required to achieve the following functions.

(1) Independent or gang control, accurate positioning and implementing acceleration/deceleration control.

(2) Optical isolation input and output I/O interface, optical isolation pulsing and direction signal output.

(3) Providing industrial standardized asynchronous serial interface to realize the communication with computers. The upper computer can be used to set and control the operation parameters of stepping motor.

(4) The positioning accuracy of stepping motor achieves $0.5 \mathrm{~mm}$, and the motion speed of platform reaches $18 \mathrm{~mm} / \mathrm{s}$.

(5) Each axis has two safety limit switches. 


\section{Hardware Design}

The design selects SCM as the processing chip of the medical device system, the reason for which is that the single chip has the advantages of small volume, great functions and wide application. The core of central processing unit is a general single chip. It has two tasks, receiving the instructions of the upper computer to process, and sending the instructions to the lower single chip, and acquiring the data and information of two single chips and processing and transferring it to the upper computer. Two lower single chips are used to control the constancy of stepping motor and box temperature.

The stepping motor motion controller designed in the paper uses single chip as core control component, which not only effectively reduces the cost. And the controller communicates with the upper computer by serial ports, which not only simplifies the connection between controller and upper computer, but also makes full uses of software resources of upper computer.

\section{Design of Central Processing Unit}

The paper selects MAX48 as the interface chip of central processing unit and lower single chip. MAX485 interface chip is a RS-485 chip in Maxim Corporation. It applies single power supply $+\mathrm{SV}$, and the rated current is $300 \mu \mathrm{A}$, and it uses half-duplex communication way. It has the function of transferring TTL level into RS-485 level. From Figure 1, we can see that the structure and pin of MAX485 is very easy, and includes a driver and receiver. RO and DI is the output of receiver and input of driver. It only needs to connect with RXD and TXD of single chip when it connects with single chip. /RE and DE is the enable pin of reception and transmission. When / RE is logic 0 , the device is in accepting state. And when DE is logic 1, the device is in transmission state. Because MAX48 is in half-duplex state, only a pin of single chip is used to control two pins. When the level of $\mathrm{A}$ is lower than that of $\mathrm{B}$, it means that the transmitted data is 0 . The wiring is easy to connect with single chip. Only the acceptation and send of a signal control MAX485 is needed. And the matched resistance is added between A and B, and the resistance about $1000 \Omega$ is generally selected. And the matched resistance in the design is $120 \Omega$.

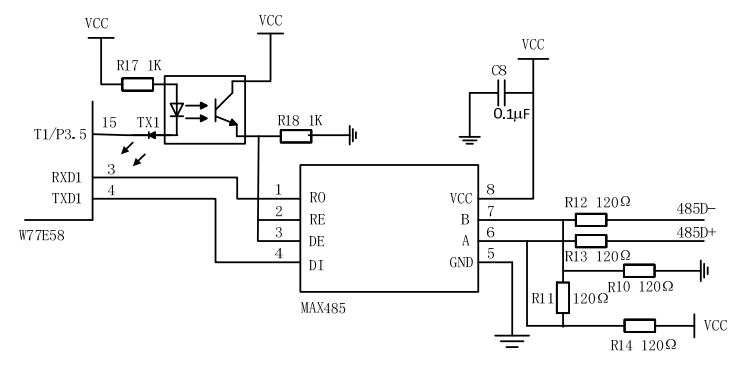

Fig. 1 Communication circuit of central processing unit and lower single chip

EEPROM circuit. EEPROM is a read-only memory which uses electric method for online erasure and reprogramming. It not only has the reading and rewriting feature of RAM in on-line operation, but also has the advantage of nonvalatile memory ROM that it still keeps the stored data after power down. It is advanced technology of embedded control solution scheme, and it is widely applied to single chip. It is used for power-down protection of data, storage of online setting parameter, table storage of users in online programming look-up table, and application of microcontroller with fewer I/O lines.

AT93C66 is a programmable read-only memory with low power consumption, low voltage and erasure electric of Atmel Corporation. It has three-wire serial interface. The capacity is $4 \mathrm{~KB}$, and it can be rewritten for 1 million times. Compared with paralleled EEPROM, AT93C66 not only can greatly save the space of printed board, but also is easy for wiring, which can be widely applied in the future.

Fig. 2 is the hardware connection of serial EEPROM chip AT93C6 and W77E58 single chip 


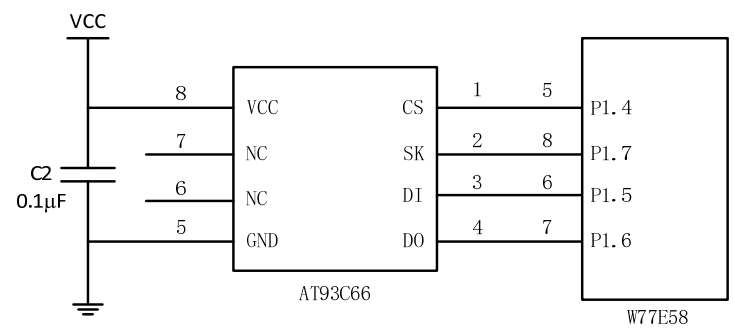

Fig. 2 Hardware connection of serial EEPROM chip AT93C6 and W77E58 single chip

W77E58 single chip has no serial bus interface of three-wire Microwave. When it connects with 93C66 with EEPROM of three-wire Microwave serial bus, the corresponding software should be used to simulate the operation of three-wire Microwave serial bus including serial clock, data input and data output. P1.4 port of W77E58 in Figure 2 is used to simulate CS of AT93C66, P1.7 port is used to simulate the clock input SK of AT93C66, and P I .5port is used to simulate the data input end DI, and P1.6 is used to simulate the data input end DO.

Detection circuit. Input circuit connects with interruption pin of single chip by voltage comparator. As shown in Figure 3, the input signals can be various such as the origin signal or limit signal of stepping motor motion. According to the variety of input signal, the single chip uses interruption processing program as the corresponding process. The detection input signal is not given in the controller. So the circuit is designed for expansion in the future.

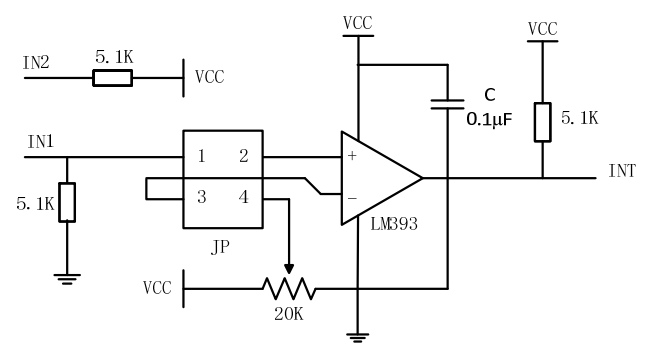

Fig. 3 Detection circuit

\section{Stepping Motor Controller}

Design of circular distributor circuit. In the system, single chip outputs four-way signals, $\mathrm{X}$-axis pulse, $\mathrm{X}$-axis direction, $\mathrm{Y}$-axis pulse and $\mathrm{Y}$-axis direction. Circular distributor PMM8713 is used to generate control pulse signal. And $\mathrm{X}$ and $\mathrm{Y}$ driver is used to drive $\mathrm{X}$-axis stepping motor and $\mathrm{Y}$-axis stepping motor, which controls objective table to move according to requirements. PMM8713 is a stepping motor pulse distributor of Japan Sanyo Electric Corporation. It has two impulse input methods, dipulse input method and single-pulse input method.

Drive circuit design. The common stepping motor drive circuit generally uses the way of chopping for constant current. The method uses the interruption of logic controller, and uses many components, so the circuit design is very complicated. The design uses UC3 842 chip to generate stepping drive signal of stepping motor, and the structure is simple and it is reliable. And it can make stepping motor reverse accurately and rapidly.

MOSFET is selected as drive switching tube, and the type is SSH8N110. MOSFET is a voltage-controlled component, so it doesn't need promotion when it drives large current, and the circuit is simple. It has the characteristics of high working frequency, rapid switching speed, small switching consumption and excellent linear region. The power MOSFET can be used in parallel. And increasing output current doesn't need averaged-flow resistance. 


\section{System Software Design}

Upper computer software. The upper computer program is written with VB language, and it can be operated by PC instrument. It not only can set the parameters of instrument, but also can read the data information.

There are two methods realizing serial communication under Windows. One method is to use APT application interface of Windows. The other method is to use serial communication control MSComm of Microsoft Visual C++ or Visual Basic. The former has widely application area, but it is very complicated and has high specialization degree, which makes it difficult in operation. The latter is easy and direct, and is convenient for serial program development. The system uses the second method to solve serial communication.

Before using serial port for data communication, a certain protocol must be used to configure. And the configuration of serial port mainly includes Baud rate, data bits, stop bits and odd-even check. The Baud rate of the program is 9600 , and the starting bit is 1 bit, the data bits is 8 , the stop bits is 1 , and there is no odd-even check.

Lower computer software. (1) Master CPU software design. Master CPU software program is the master CPU circuit board program of system. The main function is to realize the communication of sub-function modules of system and upper computer, which means to implement communication of stepping motor control system/temperature control system and upper computer. The core of master CPU control board system uses W77E58 single chip of Winbond, the reason for which is that it has two serial communications, one communication is used for 232 communication with upper computer, and the other communication is used for 485 communication with stepping motor control system/temperature control system.

(2) Stepping motor control software design. The upper computer sends the commands controlling objects to single chip by serials, and the single chip accepts and stores the commands into the cache area by the way of interruption. Then, it is compared with the coordinator of the position of the control object, and the distance of two points is computed. And the stepping motor is judged if it is forward or reversed. Designing the system firstly needs to solidify the total steps of acceleration, deceleration and constant speed of two points between different distances in ROM, which not only reduces the time of occupying CPU greatly, but also can improve the response speed of the system. When the system operates, look-up table method is used to store the acceleration, deceleration and constant speed steps into the memory.

After single chip AT89S52 accepts the instruction of upper computer, it controls the rotation direction, steps and velocity of X motor and Y motor, which achieves the objective of automatic position. The structure of stepping motor control software consists of master program, data acceptation and transmission, control code analysis and execution and speed control. Some parts are interconnected, which composes complicated motion control program. The lower computer software can implement single-axis independent control and two-axis gang control.

\section{System Debugging}

System debugging is very complicated, which should be serious to ensure reliability of system. The task is controlled around $12 \times 8$ lattice array on a X-Y plane. As shown in Figure 4, the control objects are accurately moved between any two points, and the protection switch is implemented by software, which makes it not move out of the range of lattice array. 


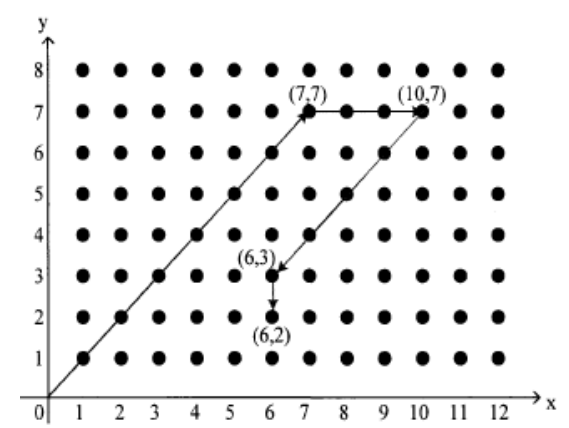

Fig. $4 \mathrm{X}-\mathrm{Y}$ coordinator plan sketch

When the circuit is tested, the program defaults the original point is $(0,0)$. Firstly, the instructions are sent to single chip from the upper computer. The single chip achieves the coordinator $(10,7)$ of destination point, which judges the steps of stepping motor in direction $\mathrm{X}$ and $\mathrm{Y}$ and directions. Then, the stepping motors in direction $\mathrm{X}$ and $\mathrm{Y}$ move with the same velocity, so the control objects move with the angle of 45 along $X$ and $Y$ axis. Unit it moves to point $(7,7)$, $\mathrm{Y}$-axis motor goes through the process of acceleration-constant high speed-deceleration-constant low speed-lock, and the movement in $\mathrm{Y}$ direction stops. And only $\mathrm{X}$ motor works, which makes control object move to $(10,7)$ along $X$ axis. When the next destination point coordinator $(6,2)$ is accepted, two motors reversed. And it firstly moves the control objects to point $(6,3)$, and lastly makes it move to the destination point $(6,2)$. The online debugging indicates that the system operates well, and accurately implements the automatic positioning functions of corotation, reversal, acceleration and deceleration and pause, which achieves the design requirements.

\section{Reference}

[1] X.G. Ding, Design and implementation of high-accuracy vector subdivision control of stepping motor, Electric Technique and Automation, 2006, 35 (4): 183-185, 188

[2] B.T. Liu, S.K. Cheng, Stepping motor and drive control system, Harbin Institute of Technology Press, 2007, 32-86.

[3] Z.G. Liu, Power electronics, Tsinghua University Press, Beijing Jiaotong University Press, 2004, 63-64.

[4] X.L. Wang, Y.H. Zhang, Research on linear adjustment and optimization of stepping motor, Micromotor, 2006, 39(8) 50-52

[5] L.X. Wang, B.Q. Wang, Applying single chip to implement variable speed control on stepping motor, Application Technique, 2003,1: 42-43.

[6] J. Xu, S.J. Ge, Applying single-chip implementation stepping motor technique to research machine tool electric appliance, 2004, 6: 25-28, 39

[7] Y.Y. Zhang, Q.M. Lai, Two-dimensional stepping motor control system based on PIC single chip, Journal of Xinyang Normal College, 2005 7: 276-278. 